This is a non-final version of an article published in final form in: Masmali, A. M., Al-Bahlal, J. M., El-Hiti, G. A., Akhtar, S., Purslow, C., Murphy, P. J., \& Almubrad, T. (2015). Repeatability and Diurnal Variation of Tear Ferning Test: Eye \& Contact Lens: Science \& Clinical Practice, 41(5), 262-267. https://doi.org/10.1097/ICL.0000000000000116

\title{
1 Repeatability and Diurnal Variation of Tear Ferning Test
}

2

3 Ali M. Masmali ${ }^{\mathrm{a}, *}$, Jarallah M. Al-Bahlal ${ }^{\mathrm{a}}$, Gamal A. El-Hiti ${ }^{\mathrm{a}}$, Saeed Akhtar ${ }^{\mathrm{a}}$, Christine

$4 \quad$ Purslow $^{\text {b }}$, Paul J. Murphy ${ }^{\mathrm{c}}$, Turki Almubrad ${ }^{\mathrm{a}}$

5

a Cornea Research Chair (CRC), Department of Optometry, College of Applied Medical

$7 \quad$ Sciences, King Saud University, P.O. Box 10219, Riyadh 11433, Saudi Arabia

$8 \quad{ }^{b}$ School of Optometry and Vision Sciences, Cardiff University, Cardiff, UK

$9{ }^{c}$ School of Optometry and Vision Science, University of Waterloo, Waterloo, Canada 10

11 The authors have no conflicts of interest to disclose.

* Corresponding author at: Cornea Research Chair (CRC), Department of Optometry, College of

Tel.: +966 11 4693547; fax: +966114693536

E-mail address: amasmali@ksu.edu.sa (A. Masmali).

Number of Tables: 2

Number of Figures: 4 
24 Objectives: To investigate tear ferning test repeatability between sessions by observing changes

25 in the tear fern pattern during the day.

27 Methods: Twenty-three healthy young adults (15 male and 8 female), ranging in age from 20 to 32 years (mean \pm SD: $22.9 \pm 3.3$ years) without signs or symptoms of dry eye disease, ocular 29 disease or contact lens wear, were enrolled in the study. Schirmer I, tear break up time (TBUT) test and McMonnies questionnaire were used to screen volunteers. Schirmer I and TBUT tests were applied to both eyes in each subject. Four samples of tear fluid were collected from the

32 right eye of each subject using glass capillaries, at set intervals during a single day (9am, 11am, 2pm and 4pm). The tear ferning (TF) patterns obtained from samples were classified according to the Masmali TF grading scale, to increments of 0.1 .

Results: The median values obtained from the McMonnies, Schirmer and TBUT tests were

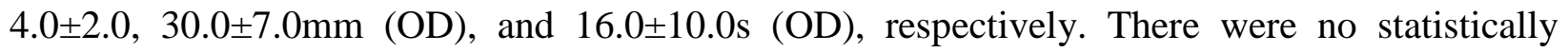
significant differences between the TF grades for tear samples collected at different times of the day (Wilks’ Lambda, $p=0.351$ ). The majority (84.8\%) of TF grades were between 0.0 and 1.5; the remaining $15.2 \%$ of subjects had TF between grades 1.6 and 1.9 . The overall mean grade for

41 the tear ferning was $1.1 \pm 0.3$. There were small, insignificant correlations between TF grades and 42 the McMonnies questionnaire $(r=0.1 .30)$ and TBUT $(r=0.248)$, and a negligible correlation 43 with Schirmer test $(\mathrm{r}=-0.046)$.

Conclusions: The results found no significant differences within the tear ferning for tear samples collected at different times of the day, suggesting that there is little diurnal variation evident. 
48 Keywords: Tear ferning; non-dry eye subjects; Masmali grading scale; Schirmer test; Tear break up time; McMonnies questionnaire

\section{INTRODUCTION}

Tear production is very important for clear vision and eye health. Dry eye patients suffer 53 from discomfort, such as sensitivity to light, stinging, burning, blurriness and grittiness, or 54 complain of scratchy and itchy eyes. ${ }^{1-3}$ The multiple causes of dry eye make its diagnoses and 55 treatment challenging. ${ }^{4}$ Moreover, the current available methods for the diagnosis of dry eye are 56 far from perfect, with poor correlations between signs and symptoms, and between diagnostic 57 tests. $^{5}$

The ideal test should be simple to use, repeatable, sensitive and specific to dry eye 59 disease, and should ideally correlate with symptoms. Several clinical tests focus on examination 60 of tear film quantity (volume), stability, or quality (composition). Tear volume assessment can be 61 carried out using the Schirmer's test ${ }^{6}$ or by tear meniscus measurement. ${ }^{7}$ The Schirmer's test is 62 the most common method for the evaluation of tear production, ${ }^{8-10}$ but its invasive approach 63 makes it liable to reflex tearing. ${ }^{11}$ The phenol red thread test (PRT) can also be used, and has 64 advantages over Schirmer's test in being more comfortable for the patient, requiring less time 65 and there is no need for anaesthesia ${ }^{8}$, but there is still a question on what exactly the thread is 66 measuring - whether it is the basal secretion rate ${ }^{12}$ or perhaps related to wetting characteristics of

67 the thread . ${ }^{13}$ Tear meniscus measurement has the advantage of being non-invasive, depending 68 on technique, but the test lacks universal cut-off values for normative data. ${ }^{2}$ 
Tear film stability can be assessed by measuring tear break-up time (TBUT). ${ }^{14}$ However,

70 further studies are needed to refine the sensitivity, specificity and reproducibility of the test. ${ }^{2}$

71 Tear clearance assessment can be evaluated by the fluorescein clearance test. ${ }^{15,16}$ The test

72 evaluates reflex tears, basal tears and tear clearance simultaneously with the advantage of being

73 relatively easy to perform and inexpensive. ${ }^{17}$ However, low specificity and sensitivity for tear

74 evaluation and reflex tears production are disadvantages. ${ }^{17,18}$ Non-invasive tear break-up time

75 (NITBUT) can assess tear stability, but it has not been confirmed whether this test is evaluating

76 changes in tear stability from changes to the lipid layer or to the overall tear film. ${ }^{19}$

77 Some aspects of the tear film chemical properties can be assessed using tear

78

79

80

81 The TearLab ${ }^{\mathrm{TM}}$ osmolarity system (TearLab ${ }^{\mathrm{TM}}$ Corp., San Diego, California) can measure the

82 osmolarity of tears efficiently, but the cost associated with the running of this test is high, and

83 repeatability requires multiple testing. ${ }^{23}$
An alternative for assessing tear film composition is to use tear ferning (TF), which has showed good specificity and sensitivity. ${ }^{24,25}$ Bodily fluids, when allowed to dry on a glass slide at room temperature and low humidity, produce ferns of specific patterns. ${ }^{26}$ The process of the TF test involves the use of a glass capillary tube to collect a sample of tears from the inferior tear meniscus. ${ }^{25,27}$ The sample is expelled from the capillary tube and the tears are allowed to dry in air at room temperature. ${ }^{26,28}$ The ferning patterns produced are then observed under light microscopy ${ }^{29}$ at magnification levels ranging from $10-100 X^{30,31}$ 
In 1984, Rolando suggested a tear ferning (TF) grading scale consisting of four types

92 (I-IV), in which Types I and II were more commonly observed in normal eye subjects, while,

93 Types III and IV were typically observed in dry eye patients. ${ }^{32}$ Recently, the Masmali 5-point TF

94 grading scale has been developed ${ }^{33}$ which overcomes some of the limitations associated with the

95 Rolando scale. ${ }^{34}$ The Masmali TF grading scale was found to have good validity in describing

96 TF patterns ${ }^{35}$, with Grades $\geq 2$ classified as abnormal. ${ }^{35,36}$ With using this new grading scale, the

97 TF test has the potential to be practiced in the clinic and can be used as a support for other dry 98 eye tests.

This paper reports on a study that investigates one aspect of the validity of the TF test: 100 testing the repeatability of tear ferning pattern during different times of the day, using the 101 Masmali grading scale.

102

103

\section{METHODS}

\section{Subjects}

Twenty-three healthy young adults (15 male and 8 female) who ranged in age from 20 to

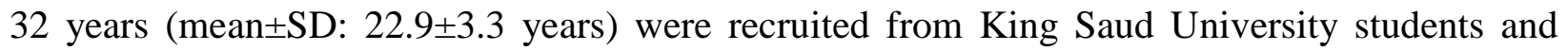

107 staff in Riyadh, Saudi Arabia. Ethical approval was obtained from the College of Applied

108 Medical Science Research Centre, King Saud University. This study followed the tenets of the

109 Declaration of Helsinki, in which informed consent was obtained from the subjects after an 110 explanation of the nature and possible consequences of the study. Subjects were then examined 111 with routine slit lamp biomicroscopy examination to assess the anterior part of the eye and to 112 confirm the absence of ocular diseases. At this point volunteers also completed the McMonnies' 113 questionnaire to exclude dry eye patients. Dry eye was diagnosed for a score $>14.5 .^{37,38}$ In 
114 addition, Schirmer I and tear break-up time (TBUT) tests were applied for both eyes of each 115 subject to assist in assessing exclusion criteria.

116

117

118

119

120

121

122

123

124

125

126

127

128

129

130

131

132

133

134

135

136

A single tear sample (first sample: 9am) was collected prior to the Schirmer test screening to avoid bias, and after applying Schirmer's test, ten minutes was allowed to expire prior to TBUT assessment. All subjects were examined in the same laboratory, where room temperature remained stable at $23^{\circ} \mathrm{C}$ and $40 \%$ humidity (one room was selected for this study and temperature and humidity were checked every day during the study). Subjects spent the day in the building at room temperature, and were examined indoors between 9am and 4pm. All tear samples were collected from the subjects by the same investigator using the same method and under the same condition.

The TearFlo ${ }^{\mathrm{TM}}$ Schirmer filter paper strips were purchased from Contacare Ophthalmics and Diagnostics (Gujarat, India) and were applied to both eyes at the same time; a value above $10 \mathrm{~mm}$ was considered as normal. The tear break-up time (TBUT) was performed three times in each eye and the average time was recorded. The cut-off value for dry eye was $<10$ seconds.

The study design was masked to avoid any bias. The McMonnies’ questionnaire, slitlamp examination, Schirmer's test and tear collections were completed by one investigator, and the imaging of the tear ferning patterns slides and the grading of the ferning patterns was completed by another investigator, who was blind to the subject's other test results.

\section{Tear collection}

The tear samples were collected at four different times during the day (9am, 11am, 2pm and $4 \mathrm{pm})$. Each sample $(1 \mu \mathrm{l})$ was collected from the lower meniscus of the right eye only using a

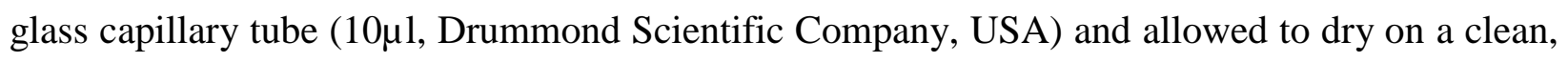


137 unused glass slide for 10 minutes under normal room temperature $\left(23^{\circ} \mathrm{C}\right)$ and humidity $(40 \%)$.

138 Samples were immediately observed under digital microscope (Olympus DP72) with 10X 139 magnification. ${ }^{35}$ Each ferning pattern observed was graded using the Masmali TF grading scale ${ }^{33}$ 140 in 0.1 increments to improve grade refinement. ${ }^{39}$

\section{Statistical Analysis}

Data were collated using Excel (Microsoft Office 2010) and analysed using SPSS

144 software (IBM Software, version 20). Data were examined for normality using Kolmogorov145 Smirnov tests and were found to be normally distributed (Kolmogorov-Smirnov, $\mathrm{p}>0.05$ ) for TF 146 grades and not normally distributed (Kolmogorov-Smirnov, $\mathrm{p}<0.05$ ) for McMonnies, Schirmer 147 and TBUT tests. The mean \pm standard deviation (SD) was used to describe the results from TF 148 grades, while the median \pm inter-quartile range (IQR) was used to describe the results for 149 McMonnies, Schirmer and TBUT tests. The parametric test (one-way repeated measures 150 ANOVA) was used to compare TF grade at different time points. Since the data collected from 151 both eyes for Schirmer and TBUT were correlated (Schirmer's test: Spearman's rho= 0.52 ; 152 TBUT: Spearman's rho $=0.74)$, the measurements for the right eye only were used. In normal eye 153 studies, it has been recommended that when the data from both eyes is highly correlated only one

154 eye per participant can be used. ${ }^{40}$ Spearman's correlation was used to investigate the relationship 155 between all data obtained (McMonnies, Schirmer, TBUT and TF grades). Correlation test was 156 used to study the relationship between TF grade, McMonnies, Schirmer and TBUT results. 157 Correlation coefficients were graded as: small (0.10 to 0.29$)$, medium ( 0.30 to 0.49$)$ and large 158 (0.50 to 1.00). ${ }^{41}$ The Coefficient of variation between the four sessions was calculated using the 159 formula (100 X SD)/overall mean). ${ }^{42,43}$ 

181 differences.

\section{RESULTS}

\section{Tear Ferning}

70

2

(1)

77

8

The median $( \pm \mathrm{IQR})$ score for the McMonnies questionnaire was $4.0 \pm 2.0$. The median $( \pm \mathrm{IQR})$ values obtained from the Schirmer and TBUT tests were $30.0 \pm 7.0 \mathrm{~mm}$ (OD) and $16.0 \pm 10.0 \mathrm{~s}(\mathrm{OD})$, respectively.

There were no significant differences between the TF grades for the four samples, collected at different sessions and different times during the day, within each subject (Wilks' Lambda, $p=0.351$ ), and there were no statistically significant differences between the pair-wise comparisons of any two samples (Table 1).

Table 1 here

The mean \pm SD TF grading pattern for the four samples collected from each subject at different times during the day is shown in Figure 1. The average coeffiecent of variation was $0.30 \%$ and the cohort range was $0.05 \%$ to $1.6 \%$.

Figure 1 here

As an example, the tear ferning patterns for the four samples collected from one subject at 9am (A), 11am (B), 2pm (C) and 4pm (D), illustrated in Figure 2, showed no significant 
The Bland-Altman plot showing the mean differences between the four sessions and the $185 \pm 2$ SD limits of agreement for all subjects is presented in Figure 3.

186

187

188

189

190

191

192

193

194

195

196

197

198

199

200

201

202

203

204

205

206

207
Figure 3 here

The tear fern grading scale results for the right eye only showed that the majority $(84.8 \%)$ of TF grades were between 0.0 and 1.5 , with the remaining $15.2 \%$ of subjects having TF grades between 1.6 and 1.9. The mean tear ferning grade for all samples collected during the day was in the range of $1.0-1.1$ (mean \pm SD: $1.1 \pm 0.3$ ), based on the Masmali TF grading scale. ${ }^{33}$ It was found that the most observed tear ferning patterns (76.1\%) corresponding to grades between 0.6 and 1.0. The TF grading scale range percentages are shown in Figure 4.

Figure 4 here

There were small, but not significant, correlations between the TF grades and the McMonnies questionnaire (Spearman; $r=0.130$ ) and TBUT (Spearman; $r=0.248$ ), and a negligible negative correlation with Schirmer test $(\mathrm{r}=-0.046)$. A medium (and significant) correlation was found between McMonnies questionnaire and Schirmer's test, with a Spearman’s correlation $(r)$ of 0.461 (Table 2).

Table 2 here 


\section{DISCUSSION}

Tear ferning has been reported to have potential to become a simple clinical test that can evaluate the quality of tear compositions. ${ }^{44}$ By drying a small tear sample on a clean glass slide

211 to produce a tear ferning pattern, aspects of tear composition, especially of electrolyte and

212 macromolecule concentration, can be observed. ${ }^{45}$ Tear ferning has its origins in examining the

213 quality of mucins from mucous secreting tissues ${ }^{44}$, but work by Rolando showed its potential for

214 assessing tear film quality. ${ }^{46}$ A significant development was the availability of the Rolando tear

215 fern scale to grade the ferning pattern produced. More recently, in response to weaknesses in the

216 design of the Rolando scale, the Masmali scale was developed. With this new scale, there is

217 potential for tear ferning to become a more regularly included test for the tear film clinician.

218 However, to make a clinical test useful, its repeatability must be known, and should be

219 within acceptable limits. Indeed, the validity of any measurement is absent when it is totally

220 unrepeatable. ${ }^{47}$ The results from this study show good repeatability, with no significant

221 differences in the TF patterns between the four tear samples collected from one eye at different

222 times in the day (9am, 11am, 2pm and 4pm), using the Masmali scale. This matches the results

223 of a previous study ${ }^{35}$ investigating repeatability with the Rolando scale, which found no

224 significant difference between tear samples collected at only two times of the day (once in the

225 morning and once in the afternoon). However, this study has improved over the previous study,

226 by having four samples for comparison (two samples at different times in the morning and two

227 samples at different times in the afternoon) rather than only two samples during the day, as well

228 as using the Masmali TF grading scale to classify the ferning patterns.

A previous study found similar levels of good repeatability, where no significant

230 difference in tear fern pattern was found between five tear samples collected from one eye over 
231 the same session, and where no significant difference was found between five drops dried from a

232 single tear sample. ${ }^{35}$ The average grade observed also matches previous results for a normal

233 cohort using the Masmali grading scale. ${ }^{36}$ The most observed grade was Grade 1 and the mean

234 was Grade 1.1.

235

Repeatability of the ferning pattern produced from a tear sample can be potentially

236 influenced by the collection method, and also by the grading scheme. ${ }^{48}$ Norn ${ }^{48}$ studied the 237 repeatability of two tear sample collection methods - the use of glass rods sampling produced 238 high variability (a coefficient of variation of 99-128\%), and while lower variability results were 239 obtained by using capillary tubes (coefficient of variation: 35\%) for sampling a random volume, 240 and (coefficient of variation: 6.4\%) for collecting a standardized tear volume, these coefficients 241 are still high. In contrast, the use of the Masmali grading scale in this study showed excellent 242 repeatability for the tear ferning test with a $0.30 \%$ average coefficient of variation.

243 This study has a limitation that it has been done only on healthy subjects, and dry eye 244 subjects may show different result. A significant diurnal variation of visual function and ocular 245 surface physiology, ${ }^{49}$ and of tear osmolarity ${ }^{50}$ have been found in dry eye subjects. So it could be 246 assumed that variation in a dry eye cohort may produce some variability and so the next study 247 that needs doing is to repeat this one using a cohort of dry eye subjects. This study also used 248 fluorescein BUT, and non-invasive TBUT would reveal different characteristics of the tear film, 249 which might be helpful in assessing correlation of tear ferning with other clinical tests for dry 250 eye.

The results from this study show that tear ferning has good repeatability, and that the use 252 of the Masmali grading scale, in a healthy subject cohort, will produce consistent grading results. 253 It has also shown that a tear sample collected a different time points will produce a similar 
254 ferning pattern. These results support the tear ferning test and suggest that it has potential for 255 clinical and research use, as part of a routine tear film examination.

\section{ACKNOWLEDGEMENTS}

The authors extend their appreciation to the College of Applied Medical Sciences 260 Research Center and the Deanship of Scientific Research at King Saud University for its funding 261 of this research.

\section{REFERENCES}

264 1. Jumblatt MM, McKenzie RW, Steele PS, Emberts CG, Jumblatt JE. MUC7 expression in 265 the human lacrimal gland and conjunctiva. Cornea 2003; 22:41-45.

266 2. Abelson MB, Ousler G 3rd. The pros and cons of dry-eye test. Rev Ophthalmol 2011; $267 \quad 7: 62-65$.

268 3. Abelson MB, Ousler G 3rd, Nally LA, Emory TB. Dry eye syndromes: diagnosis, clinical 269

270 trials and pharmaceutical treatment - “improving clinical trials”. Adv Exp Med Biol 2002;

271 4. Kent C. Dry eye diagnosis: 21st - Century tools. Rev Ophthalmol 2013; 13:28-41.

272 5. Savini G, Prabhawsat P, Kojima T, Grueterich M, Espana E, Goto E. The challenge of 273 dry eye diagnosis. Clin Ophthalmol 2008; 2:31-55.

274 6. Schirmer O. Studien zur physiologie und pathologie der tranen-absonderung und 275 tranenabfuhr. Graefes Arch Clin Exp Ophthalmol 1903; 56:197-291.

276 7. Tiffany JM. Surface tension in tears. Arch Soc Exp Ophthalmol 2006; 81:363-366. 
277 8. Masmali A, Alqahtani TA, Alharbi A, El-Hiti GA. Comparative study of repeatability of phenol red thread test versus Schirmer's test in normal adults in Saudi Arabia. Eye Contact Lens 2014; 40:127-131.

280

9. $\quad$ Bawazeer AM, Hodge WG. One-minute Schirmer test with anesthesia. Cornea 2003;

281 22:285-287.

282 10. de Monchy I, Gendron G, Miceli C, Pogorzalek, N, Mariette X, Labetoulle M. 283

11. Cho P, Yap M. Schirmer test I. A review. Optom Vis Sci 1993; 70:152-156.

287 12. Sakamoto R, Bennett ES, Henry VA, et al. The phenol red thread tear test: a cross288

13. Tomlinson A, Blades KJ, Pearce EI. What does the phenol red thread test actually cultural study. Invest Ophthalmol Vis Sci 1993;34:3510-3514.

29114 Lemp MA. Breakup of the tear film. Int Ophthalmol Clin 1973; 13:97-102.

292 15. Prabhasawat P, Tseng SCG. Frequent association of delayed tear clearance in ocular

16. Pflugfelder SC, Tseng SCG, Sanabria O, Kell H, Garcia, CG, Felix C, Feuer W, Reis BL. Evaluation of subjective assessments tear-film disorders known to cause ocular irritation.

297 17. Jordan A, Baum J. Basic tear flow. Does it exit? Ophthalmology 1980; 87:920-930.

298 18. Foulks GN. Challenges and pitfalls in clinical trials of treatments for dry eye. Ocul Surf 299 2003; $1,20-30$. 
19. 2007 Report of the International Dry Eye WorkShop (DEWS). Ocul Surf 2007; 5(2).

20. Lemp MA, Bron, AJ, Baudouin C, Benítez Del Castilo JM, Geffen D, Tauber J, Foulks GN, Pepose JS, Sullivan D. Tear osmolarity in the diagnosis and management of dry eye disease. Am J Ophthalmol 2011; 151:792-799.

21. Szalai E, Berta A, Szekanecz Z, Szûcs G, Módis L. Evaluation of tear osmolarity in nonSjögren and Sjögren syndrome dry eye patients with the TearLab system. Cornea 2012; 31:867-871.

22. Masmali A, Alrabiah S, Alharbi A, El-Hiti GA, Almubrad T. Investigation of tear osmolarity using the TearLab ${ }^{\mathrm{TM}}$ osmolarity system in normal adults in Saudi Arabia. Eye Contact Lens 2014; 40:74-78.

23. Khanal S, Millar TJ. Barriers to clinical uptake of tear osmolarity measurments. $B r J$ Ophthalmol 2012; 96:341-344.

24. Maragou M, Vaikousis E, Ntre A, Koronis N, Georgiou P, Hatzidimitriou M, Sotsiou F, Dantis P. Tear and saliva ferning tests in Sjogren’s syndrome (SS). Clin Rheumatol 1996; 15:125-132.

25. Masmali A, Purslow C, Murphy PJ. The Tear ferning test: a simple clinical technique to evaluate the ocular tear film. Clin Exp Optom 2014; 97:399-406.

26. Abou-Shabanah EH, Plotz EJ. A biochemical study of the cervical and mucus fern phenomenon. Am J Obstet Gynecol 1957; 74:559-568.

27. Norn M. Quantitative tear ferning. Clinical investigations. Acta Ophthalmol. 1994; 72:369-372.

28. Kogbe O, Liotet S, Tiffany JM. Factors responsible for tear ferning. Cornea 1991; 10:433-444. 
323 29. Golding TR, Brennan NA. The basis of tear ferning. Clin Exp Optom 1989; 72:102-112.

324 30. Norn M. Ferning in conjunctival-cyctologic preparations. Crystallisation in stained semiquantitative pipette samples of conjunctival fluid. Acta Ophthalmol 1987; 66:201-

327 31. Ravazzoni L, Ghini C, Macri A, Rolando M. Forecasting of hydrophilic contact lens

32. Rolando M. Tear mucus ferning test in normal and keratoconjunctivitis sicca eyes.

33. Masmali AM, Murphy PJ, Purslow C. Development of a new grading scale for tear 333 ferning. Cont Lens Anterior Eye 2014; 37:178-184.

334 34. Tabbara KF, Okumoto M. Ocular ferning test. A qualitative test for mucus deficiency. 335 336

36. Masmali AM, Al-Qhtani S, Al-Gasham TM, El-Hiti GA, Purslow C, Murphy PJ. Ophthalmology 1982; 89:712-714.

35. Masmali AM. Development of a tear ferning test protocol and a new grading scale. $\mathrm{PhD}$ thesis, Cardiff University, 2010.

341 37. McMonnies CW, Ho A. Responses to a dry eye questionnaire from a normal population. Application of a new grading scale for tear ferning in healthy and dry eye subjects. Cont Lens Anterior Eye 2014; in press; doi: 10.1016/j.clae.2014.09.007.

$343 \quad 38 . \quad$ Nichols KK, Nichols JJ, Mitchell G, Lynn M. The reliability and validity of McMonnies $344 \quad$ dry eye index. Cornea 2004; 23:365-371. 
345 39. Bailey IL, Bullimore MA, Raasch TW, Taylor HR. Clinical grading and the effects of scaling. Invest Ophthalmol Vis Sci 1991; 32:422-432.

347 40. McAlinden C, Khadka J, Pesudovs K. Statistical methods for conducting agreement

41. Cohen JW. Statistical power analysis for the behavioral sciences. Hillsdale NJ, Lawrence Erlbaum Associates, 1988.

42. Li H, Leung, CKS, Cheung CYL, Wong L, Pang CP, Weinred RN, Lam DSC. Repeatability and reproducibility of anterior chamber angle measurement with anterior segment optical coherence tomography. Br J Ophthalmol 2007; 91:1490-1492.

43. Polito A, Del Berrello M, Zemella N, Bandello F. Repeatability and reproducibility of 356 fast macular thickness mapping with stratus optical coherence tomography. Arch Ophthalmol 2005; 123:1330-1337.

44. Tabbara KF, Okumoto M. Ocular ferning test. A qualitative test for mucus deficiency. Ophthalmology 1982; 89: 712-714.

360 45. Kogbe O, Liotet S, Tiffany JM. Factors responsible for tear ferning. Cornea 1991; 10 : $433-444$.

362 46. Rolando M. Tear mucus ferning test in normal and keratoconjunctivitis sicca eyes. Chibret Int J Ophthalmol 1984; 2: 32-41.

47. Chinn S. Statistics in respiratory medicine. 2. Repeatability and method comparison. Thorax 1991; 46:454-456.

366 48. Norn M. Quantitative tear ferning. Methodologic and experimental investigations. Acta 367 Ophthalmol 1988; 66:201-205. 

380
49. Walker PM, Lane KL, Ousler GW, 3rd, Abelson MB. Diurnal variation of visual function and the signs and symptoms of dry eye. Cornea 2010; 29:607-612.

50. Li M, Du C, Zhu D, Shen M, Cui L, Wang J. Daytime variations of tear osmolarity and tear meniscus volume. Eye Contact Lens 2012; 38:282-287.

\section{Figures Legend}

FIG. 1. The mean \pm SD TF grade for the four samples collected from each subject at different time during the day.

FIG. 2. Tear ferning patterns of the four samples collected from one subject at 9am (A), 11am (B), 2pm (C) and 4pm (D), showing no significant differences (Grade 0).

FIG. 3. Bland-Altman plot showing the mean differences between the four sessions and the \pm 2 SD limits of agreement for all subjects.

FIG. 4. Percentages of the TF grades range during the day. 
TABLE 1. Mean Differences and Confidence Interval for Repeatability of TF Grades

\begin{tabular}{|c|c|c|c|c|c|}
\hline \multirow{3}{*}{\multicolumn{2}{|c|}{$\begin{array}{l}\text { Tear } \\
\text { Samples }\end{array}$}} & \multirow{3}{*}{$\begin{array}{c}\text { Mean } \\
\text { Differences }\end{array}$} & \multirow[t]{3}{*}{ Sig. } & \multicolumn{2}{|c|}{ 95\% Confidence Interval of th894 } \\
\hline & & & & \multicolumn{2}{|c|}{ Differences } \\
\hline & & & & Lower & Upper \\
\hline \multirow{3}{*}{1} & 2 & -0.004 & 1 & -0.266 & 0.25896 \\
\hline & 3 & 0.035 & 1 & -0.184 & 0.253 \\
\hline & 4 & 0.135 & 0.797 & -0.115 & 0.38397 \\
\hline \multirow{3}{*}{2} & 1 & 0.004 & 1 & -0.258 & 0.266 \\
\hline & 3 & 0.039 & 1 & -0.234 & 0.31398 \\
\hline & 4 & 0.139 & 0.598 & -0.095 & 0.374 \\
\hline \multirow{3}{*}{3} & 1 & -0.035 & 1 & -0.253 & $0.184^{29}$ \\
\hline & 2 & -0.039 & 1 & -0.312 & 0.234 \\
\hline & 4 & 0.100 & 1 & -0.160 & 0.360 \\
\hline \multirow{3}{*}{4} & 1 & -0.135 & 0.797 & -0.385 & $0.11_{401}$ \\
\hline & 2 & -0.139 & 0.598 & -0.374 & 0.095 \\
\hline & 3 & -0.100 & 1 & -0.360 & 0.16402 \\
\hline
\end{tabular}

TABLE 2. Correlation Between TF Grade, McMonnies Score, Schirmer and TBUT Tests

405

\begin{tabular}{llcccc}
\hline Test/Correlation & TF & McMonnies & Schirmer & TBUT \\
\hline \multirow{2}{*}{ TF } & Spearman's Correlation & 1 & 0.130 & -0.046 & 0.248 \\
& Sig. (2-tailed) & - & 0.553 & 0.834 & 0.254 \\
& N & 23 & 23 & 23 & 23 \\
McMonnies & Spearman's Correlation & 0.130 & 1 & $0.461^{a}$ & -0.183 \\
& Sig. (2-tailed) & 0.553 & - & 0.027 & 0.403 \\
& N & 23 & 23 & 23 & 23 \\
\multirow{5}{*}{ Schirmer (OD) } & Spearman's Correlation & -0.046 & $0.461^{a}$ & 1 & -0.189 \\
& Sig. (2-tailed) & 0.834 & $\mathbf{0 . 0 2 7}$ & - & 0.389 \\
& N & 23 & 23 & 23 & 23 \\
\multirow{5}{*}{ TBUT (OD) } & Spearman's Correlation & 0.248 & -0.183 & -0.189 & 1 \\
& Sig. (2-tailed) & 0.254 & 0.403 & 0.389 & - \\
& N & 23 & 23 & 23 & 23 \\
\hline
\end{tabular}

406

407

${ }^{a}$ Correlation is significant at the 0.05 level.

408

409 


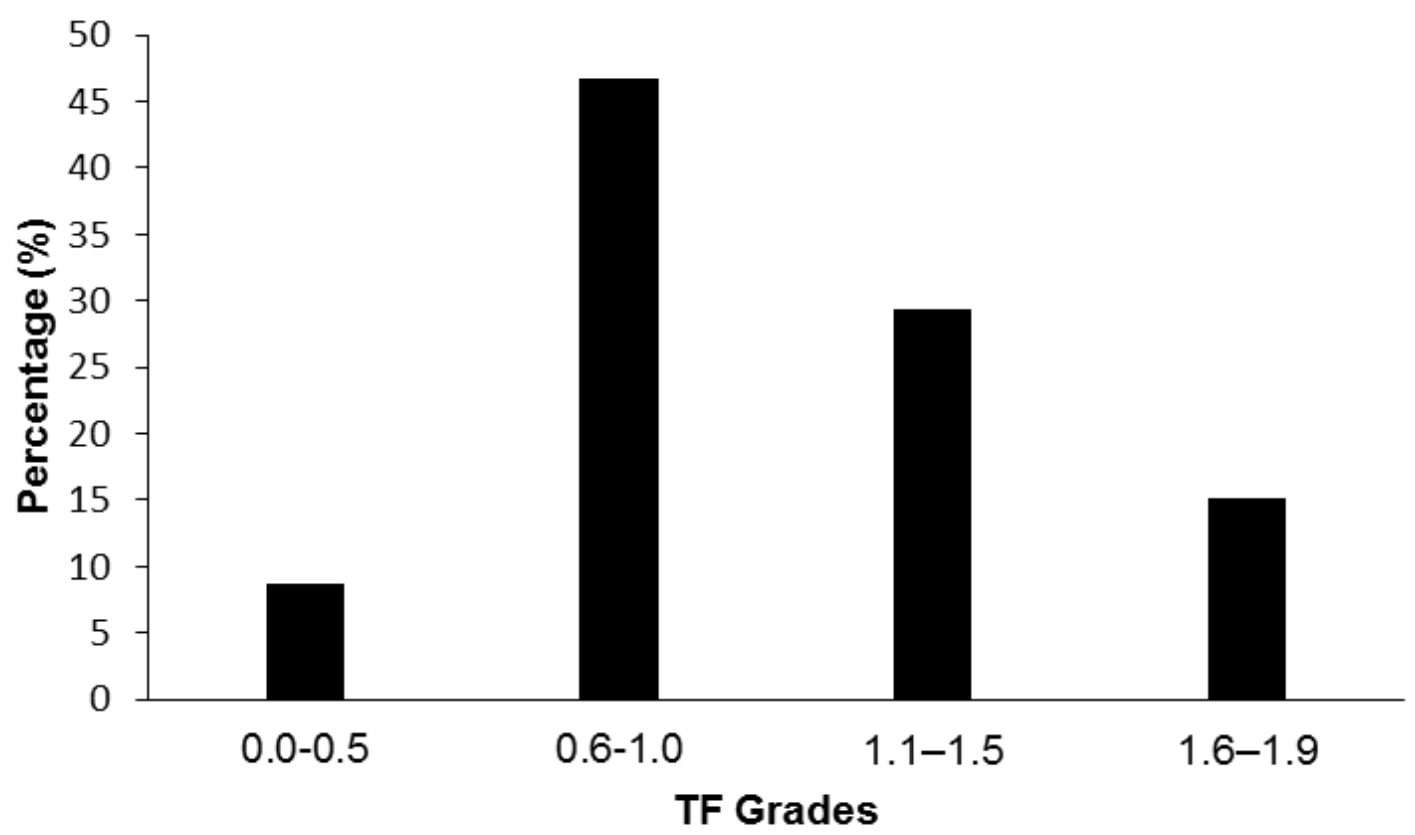

411

Figure 4 


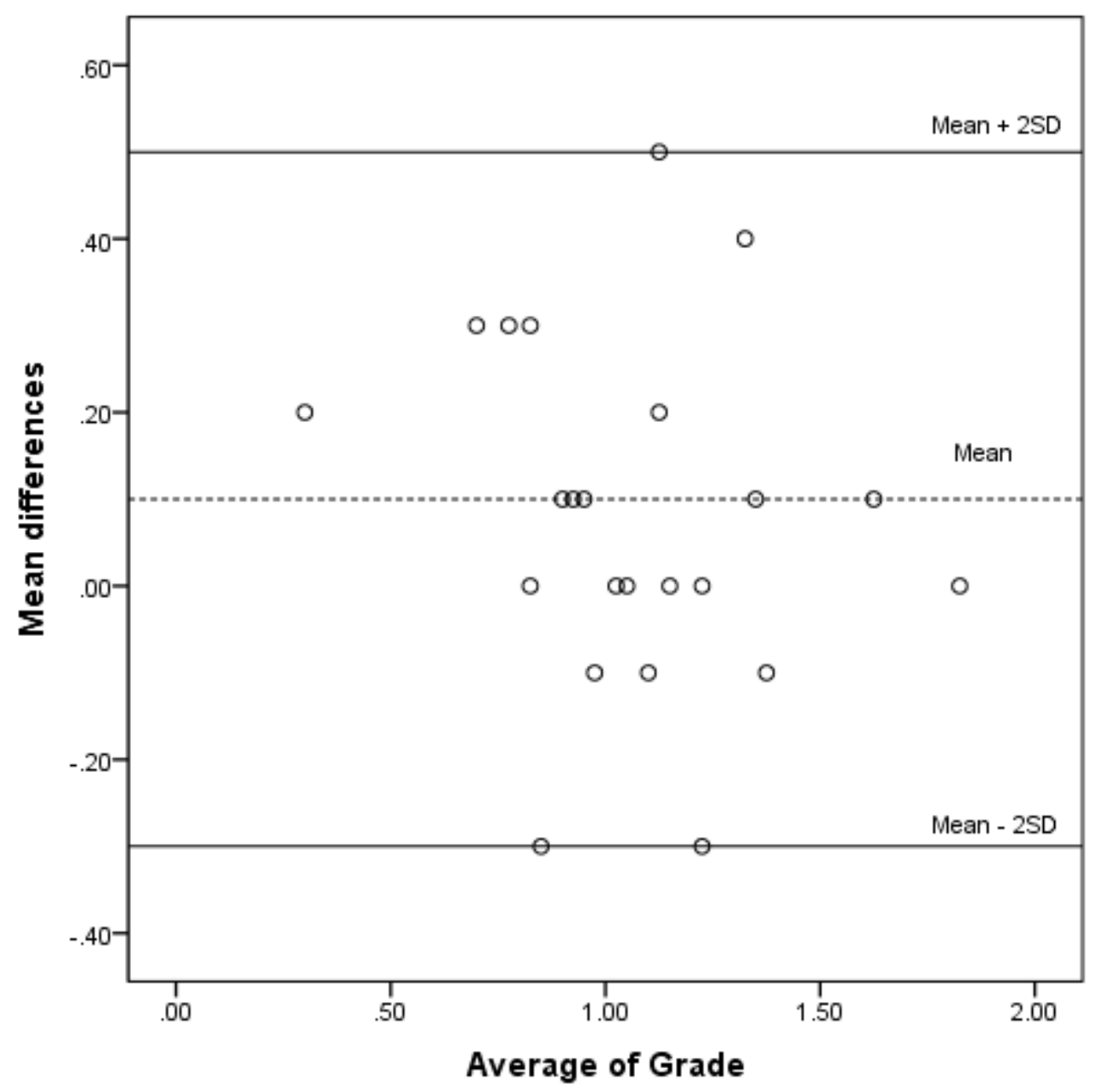

413

414

415

Figure 3 


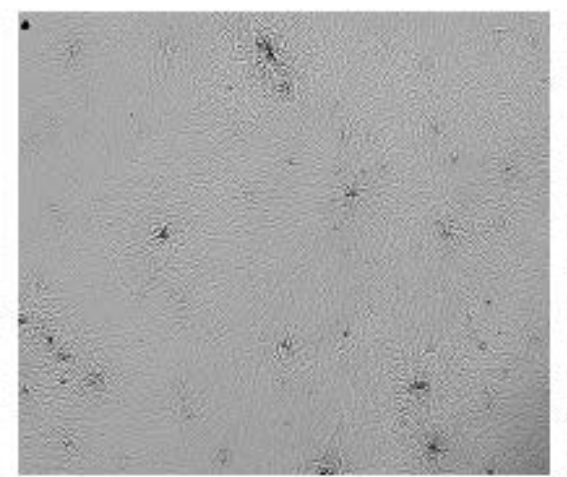

A (9am)

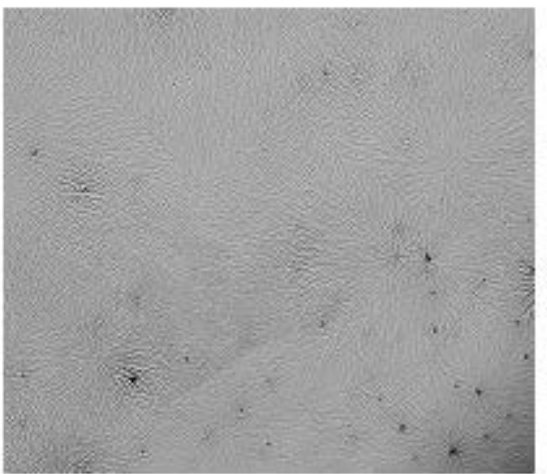

C (2pm)

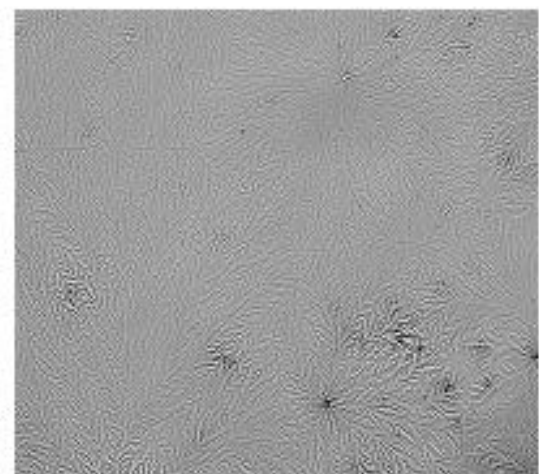

B (11am)

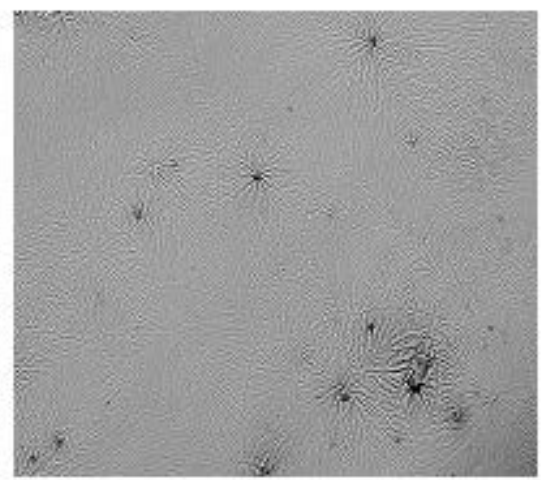

D (4pm)

Figure 2

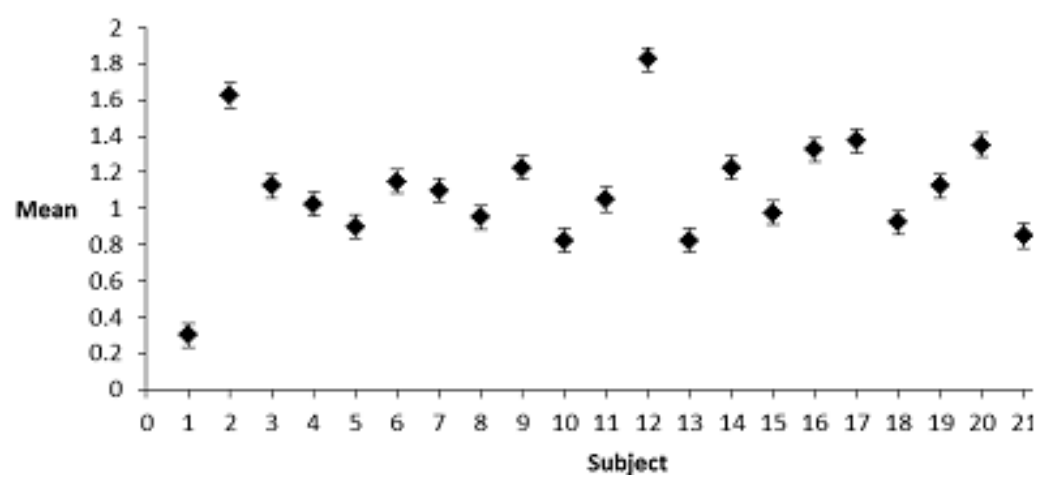

